Pacific Journal of Mathematic 


\title{
ON A CLASS OF UNBOUNDED OPERATOR ALGEBRAS II
}

\author{
Atsushi Inoue
}

In this paper we continue our study of unbounded operator algebras. On the basis of the space $L^{\omega}[0,1]$ introduced by $\mathbf{R}$. Arens [1] we define and investigate unbounded Hilbert algebras. The primary purpose of this paper is to investigate the relation between unbounded Hilbert algebras and $E W^{*}$-algebras and the structure of some $E W^{*}$-algebras.

1. Introduction. In a previous paper [10] we began our study of $E W^{*}$-algebras. For the definitions and the basic properties concerning $E W^{*}$-algebras is referred to [10]. It is well known that semifinite von Neumann algebras are related to Hilbert algebras. That is, if $\mathscr{D}_{0}$ is a Hilbert algebra, then the left von Neumann algebra $\mathcal{U}_{0}\left(\mathscr{D}_{0}\right)$ is defined and $\mathcal{U}_{0}\left(\mathscr{D}_{0}\right)$ is a semifinite von Neumann algebra and conversely if $\mathfrak{U}$ is a semifinite von Neumann algebra, then there exists a Hilbert algebra $\mathscr{D}_{0}$ such that $\mathfrak{A}$ is isomorphic to the left von Neumann algebra $\mathscr{U}_{0}\left(\mathscr{D}_{0}\right)$. In this paper we study the above facts about $E W^{*}$-algebras. So, our starting point will be the extension of Hilbert algebras.

DEFINITION 1.1. Let $\mathscr{D}$ be a pre-Hilbert space with inner product ( | ) and a $*$-algebra. If $\mathscr{D}$ satisfies the following conditions (1) $\sim(3)$;

(1) $(\xi \mid \eta)=\left(\eta^{*} \mid \xi^{*}\right), \quad \xi, \eta \in \mathscr{D}$

(2) $(\xi \eta \mid \zeta)=\left(\eta \mid \xi^{*} \zeta\right), \quad \xi, \eta, \zeta \in \mathscr{D}$

By (2) we define $\pi(\xi)$ and $\pi^{\prime}(\eta)$ by;

$$
\pi(\xi) \eta=\pi^{\prime}(\eta) \xi=\xi \eta, \quad \xi, \eta \in \mathscr{D} .
$$

Then $\pi(\xi)$ and $\pi^{\prime}(\eta)$ are closable operators on $\mathscr{D}$ and we have $\pi(\xi)^{*} \supset \pi\left(\xi^{*}\right)$ and $\pi^{\prime}(\eta)^{*} \supset \pi^{\prime}\left(\eta^{*}\right)$. We call $\pi$ (resp. $\pi^{\prime}$ ) the left (resp. right) regular representation of $\mathscr{D}$.

(3) Putting

$$
\mathscr{D}_{0}=\{\xi \in \mathscr{D} ; \pi(\xi) \text { is continuous }\}
$$

$\mathscr{D}_{0}^{2}$ is dense in $\mathscr{D}$, then $\mathscr{D}$ is called an unbounded Hilbert algebra over $\mathscr{D}_{0}$. In particular, if $\mathscr{D}_{0} \neq \mathscr{D}$, then $\mathscr{D}$ is called a pure unbounded Hilbert algebra over $\mathscr{D}_{0}$.

In $\S 2$ we investigate the properties of unbounded Hilbert algebras and we introduce examples of such unbounded Hilbert algebras $\left(L^{\omega}[0,1]\right.$, 
$L^{\omega}(-\infty, \infty), L_{1 *}^{\omega}(-\infty, \infty), L_{2}^{\omega}(-\infty, \infty), L_{1}^{\omega}(G), L_{2}^{\omega}(G)(G ;$ unimodular locally compact group)).

In $\$ 3$ we consider the noncommutative integration with respect to a von Neumann algebra as constructed by Segal in [14]. Let $\mathscr{D}$ be a pure unbounded Hilbert algebra over $\mathscr{D}_{0}$. Then $L^{\omega}\left(\mathscr{D}_{0}\right)$ and $L_{2}^{\omega}\left(\mathscr{D}_{0}\right)$ are defined and they are pure unbounded Hilbert algebras. In particular, $L_{2}^{\omega}\left(\mathscr{D}_{0}\right)$ is maximal in pure unbounded Hilbert algebras containing $\mathscr{D}_{0}$. Furthermore $\mathscr{D}^{2}$ (resp. $\mathscr{D}$ ) is a $*$-subalgebra of pure unbounded Hilbert algebra $L^{\omega}\left(\mathscr{D}_{0}\right)\left(\right.$ resp. $\left.L_{2}^{\omega}\left(\mathscr{D}_{0}\right)\right)$ (Theorem 3.9.). We can define a left $E W^{*}$-algebra $U(\mathscr{D})$ of a pure unbounded Hilbert algebra $\mathscr{D}$ over $\mathscr{D}_{0}$, i.e., $U(\mathscr{D})$ is a minimal $E W^{*}$-algebra on $L_{2}^{\omega}\left(\mathscr{D}_{0}\right)$ over $U_{0}\left(\mathscr{D}_{0}\right)$ and $\overline{U(\mathscr{D})} \supset \overline{\pi(\mathscr{D})}$, where we denote by $\bar{A}$ the smallest closed extension of a closable operator $A$ and we put $\overline{\mathfrak{U}}=\{\bar{A} ; A \in \mathfrak{U}\}$ (Theorem 3.10.).

In $\S 4$ we define traces on $E W^{*}$-algebras and we investigate the structure of some $E W^{*}$-algebras.

Definition 1.2. Let $\mathfrak{A}$ be an $E W^{*}$-algebra and let $\varphi$ be a map of $\mathfrak{P}^{+}$into $[0, \infty]$. If $\varphi$ satisfies the following conditions $(1) \sim(3)$, then $\varphi$ is called a trace on $\mathfrak{A}^{+}$;

(1) $\varphi(S+T)=\varphi(S)+\varphi(T)$

(2) $\varphi(\lambda S)=\lambda \varphi(S)$

$S, T \in \mathfrak{U}^{+}$

(3) $\varphi\left(S^{*} S\right)=\varphi\left(S S^{*}\right)$,

$\lambda \geqq 0, S \in \mathfrak{Q}^{+}$ $S \in \mathfrak{A}$.

If the conditions $\varphi(S)=0, S \in \mathfrak{U}^{+}$implies $S=0$, then $\varphi$ is called faithful. If, for each increasing net $\left\{T_{\alpha}\right\}$ of $\mathfrak{U}^{+}$that converges $\sigma$-weakly to $S \in \mathfrak{I}^{+}$(hereafter we denote $T_{\alpha} \uparrow S$ ), we have $\varphi\left(T_{\alpha}\right) \uparrow \varphi(S)$, then $\varphi$ is called normal. If $\varphi(S)<\infty$ for every $S \in \mathfrak{Q}^{+}$, then $\varphi$ is called finite. If, for each $S \in \mathfrak{Q}^{+}$, there exists a net $\left\{T_{\alpha}\right\}$ such that $T_{\alpha} \uparrow S$ and $\varphi\left(T_{\alpha}\right)<\infty$, then $\varphi$ is called semifinite.

Let $U(\mathscr{D})$ be the left $E W^{*}$-algebra of a pure unbounded Hilbert algebra $\mathscr{D}$ over $\mathscr{D}_{0}$. Then there exists a faithful normal semifinite trace $\varphi$ on $U(\mathscr{D})^{+}$such that $\varphi / \mathcal{U}(\mathscr{D})_{b}^{+}$equals the natural trace on $\mathscr{U}_{0}\left(\mathscr{D}_{0}\right)^{+}$and $\mathcal{U}(\mathscr{D})\left(\mathfrak{N}_{\varphi}\right)_{b} \subset \mathfrak{N}_{\varphi}$ (we note $\mathfrak{N}_{\varphi}=\left\{T \in \mathscr{U}(\mathscr{D}) ; \varphi\left(T^{*} T\right)<\infty\right\}$ and $\left(\mathfrak{N}_{\varphi}\right)_{b}=$ $\mathfrak{N}_{\varphi} \cap \mathcal{U}(\mathscr{D})_{b}$ ) (Theorem 4.2.). Conversely if $\mathfrak{A}$ is an $E W^{*}$-algebra with a faithful normal semifinite trace $\varphi$ satisfying $\mathfrak{A}\left(\mathfrak{N}_{\varphi}\right)_{b} \subset \mathfrak{N}_{\varphi}$, then $\mathfrak{N}_{\varphi}$ is a pure unbounded Hilbert algebra over $\left(\mathfrak{N}_{\varphi}\right)_{b}$ and $\mathfrak{N}$ is isomorphic to the left $E W^{*}$-algebra $U\left(\mathfrak{N}_{\varphi}\right)$ of $\mathfrak{N}_{\varphi}$ (Theorem 4.11.).

2. Unbounded Hilbert algebras. In this section let $\mathscr{D}$ be a pure unbounded Hilbert algebra over $\mathscr{D}_{0}$ and let $\mathfrak{S}$ be the completion of $\mathscr{D}$. Clearly $\mathscr{D}_{0}$ is a Hilbert algebra and the completion of $\mathscr{D}_{0}$ is a Hilbert space $\mathfrak{S}$. For each $x \in \mathfrak{H}$ we define $\pi_{0}(x)$ and $\pi_{0}^{\prime}(x)$ by;

$$
\begin{array}{ll}
\pi_{0}(x) \xi=\overline{\pi_{0}^{\prime}(\xi)} x, & \xi \in \mathscr{D}_{0} \\
\pi_{0}^{\prime}(x) \xi=\overline{\pi_{0}(\xi)} x, & \xi \in \mathscr{D}_{0},
\end{array}
$$


where $\pi_{0}$ (resp. $\left.\pi_{0}^{\prime}\right)$ is the left (resp. right) regular representation of the Hilbert algebra $\mathfrak{D}_{0}$. Then $\pi_{0}(x)$ and $\pi_{0}^{\prime}(x)$ are linear operators on $\mathfrak{S}$ with domain $\mathscr{D}_{0}$. By ([12] Theorem 3) we have

$$
\overline{\pi_{0}(J x)}=\pi_{0}(x)^{*}, \quad \overline{\pi_{0}^{\prime}(J x)}=\pi_{0}^{\prime}(x)^{*}
$$

for all $x \in \mathfrak{H}$, where $J$ denotes the involution of $\mathfrak{S}$.

LEMma 2.1. For each $\xi \in \mathscr{D}$ we have

(1) $\overline{\pi(\xi)}=\overline{\pi_{0}(\xi)}, \overline{\pi^{\prime}(\xi)}=\overline{\pi_{0}^{\prime}(\xi)}$;

(2) $\overline{\pi\left(\xi^{*}\right)}=\pi(\xi)^{*}, \overline{\pi^{\prime}\left(\xi^{*}\right)}=\pi^{\prime}(\xi)^{*}$.

Proof. (1); Clearly we get $\pi_{0}(\xi) \subset \pi(\xi)$. Hence $\pi_{0}(\xi)^{*} \supset$ $\pi(\xi)^{*}$. Since $\pi_{0}(\xi)^{*}=\overline{\pi_{0}\left(\xi^{*}\right)}$ and $\pi(\xi)^{*} \supset \pi\left(\xi^{*}\right)$, we have

$$
\overline{\pi_{0}(\xi)}=\pi_{0}\left(\xi^{*}\right)^{*} \supset \pi\left(\xi^{*}\right)^{*} \supset \overline{\pi(\xi)} .
$$

Therefore we get $\overline{\pi_{0}(\xi)}=\overline{\pi(\xi)}$.

(2); By (1) we have

$$
\overline{\pi\left(\xi^{*}\right)}=\overline{\pi_{0}\left(\xi^{*}\right)}=\pi_{0}(\xi)^{*}=\pi(\xi)^{*}
$$

LEMMA 2.2. For each $\lambda, \mu \in \mathcal{S}$ (the field of complex numbers) and $\xi, \xi_{1}, \eta, \eta_{1} \in \mathscr{D}(i=1,2)$ we have

$$
\begin{aligned}
& \pi\left(\lambda \xi_{1}+\mu \xi_{2}\right)=\lambda \pi\left(\xi_{1}\right)+\mu \pi\left(\xi_{2}\right) \\
& \pi\left(\xi_{1} \xi_{2}\right)=\pi\left(\xi_{1}\right) \pi\left(\xi_{2}\right) \\
& \pi\left(\xi^{*}\right) \subset \pi(\xi)^{*} \\
& \pi^{\prime}\left(\lambda \eta_{1}+\mu \eta_{2}\right)=\lambda \pi^{\prime}\left(\eta_{1}\right)+\mu \pi^{\prime}\left(\eta_{2}\right) \\
& \pi^{\prime}\left(\eta_{1} \eta_{2}\right)=\pi^{\prime}\left(\eta_{2}\right) \pi^{\prime}\left(\eta_{1}\right) \\
& \pi^{\prime}\left(\eta^{*}\right) \subset \pi^{\prime}(\eta)^{*}
\end{aligned}
$$

Putting

$$
\pi(\xi)^{*}=\pi\left(\xi^{*}\right), \quad \pi^{\prime}(\eta)^{*}=\pi^{\prime}\left(\eta^{*}\right),
$$

$\pi(\mathscr{D})$ and $\pi^{\prime}(\mathscr{D})$ are \#-algebras on $\mathscr{D}$ and we have the following properties;

(1) $\pi(\mathscr{D})_{b}=\pi\left(\mathscr{D}_{0}\right), \quad \pi^{\prime}(\mathscr{D})_{b}=\pi^{\prime}\left(\mathscr{D}_{0}\right)$ 
(2) $J \pi(\xi) J=\pi^{\prime}(\xi)^{*}, \quad J \pi^{\prime}(\xi) J=\pi(\xi)^{*}, \quad \xi \in \mathscr{D}$;

(3) $\pi(\xi) \pi^{\prime}(\eta)=\pi^{\prime}(\eta) \pi(\xi), \quad \xi, \eta \in \mathscr{D}$;

(4) $\overline{\pi(\xi)^{*}}=\pi(\xi)^{*}, \quad \overline{\pi^{\prime}(\xi)^{*}}=\pi^{\prime}(\xi)^{*}, \quad \xi \in \mathscr{D}$.

Hence we get

$$
\overline{\pi(\mathscr{D})_{b}^{\prime \prime \prime}}=\mathscr{U}_{0}\left(\mathscr{D}_{0}\right), \quad \overline{\pi^{\prime}(\mathscr{D})_{b}^{\prime \prime \prime}}=\mathscr{V}_{0}\left(\mathscr{D}_{0}\right)
$$

where $\mathscr{U}_{0}\left(\mathscr{D}_{0}\right)$ (resp. $\left.\mathscr{V}_{0}\left(\mathscr{D}_{0}\right)\right)$ is the left (resp. right) von Neumann algebra of $\mathscr{D}_{0}$.

Proposition 2.3. For each $\lambda \in \mathbb{E}$ and $\xi, \eta \in \mathscr{D}$ we have

$$
\begin{aligned}
& \overline{\pi(\xi)}+\overline{\pi(\eta)}=\overline{\pi(\xi+\eta)}, \quad \overline{\pi(\xi)} \cdot \overline{\pi(\eta)}=\overline{\pi(\xi \eta)}, \\
& \lambda \cdot \overline{\pi(\xi)}=\overline{\pi(\lambda \xi)}, \quad \overline{\pi(\xi)^{*}}=\overline{\pi\left(\xi^{*}\right)} .
\end{aligned}
$$

Therefore $\overline{\pi(\mathscr{D})}$ is a *-algebra of closed operators on $\mathfrak{H}$ under the operations of strong sum, strong product, adjoint and strong scalar multiplication. Similarly $\overline{\pi^{\prime}(\mathscr{D})}$ is a *-algebra of closed operators on 5. Furthermore we have

$$
\overline{J \pi(\xi)} J=\pi^{\prime}(\xi)^{*}, \quad \overline{J^{\prime}(\xi)} J=\pi(\xi)^{*}, \quad \xi \in \mathscr{D} .
$$

Proof. By Lemma 2.1. we have $\overline{\pi(\xi)}=\pi\left(\xi^{*}\right)^{*}$ for every $\xi \in \mathscr{D}$ and hence

$$
\begin{aligned}
\overline{\pi(\xi)}+\overline{\pi(\eta)} & =\overline{\overline{\pi(\xi)}+\overline{\pi(\eta)}}=\overline{\pi\left(\xi^{*}\right)^{*}+\pi\left(\eta^{*}\right)^{*}} \\
& \subset\left(\pi\left(\xi^{*}\right)+\pi\left(\eta^{*}\right)\right)^{*}=\pi\left((\xi+\eta)^{*}\right)^{*} \\
& =\overline{\pi(\xi+\eta)}
\end{aligned}
$$

and so $\overline{\pi(\xi)}+\overline{\pi(\eta)}=\overline{\pi(\xi+\eta)}$. Similarly $\overline{\pi(\xi)} \cdot \overline{\pi(\eta)}=\overline{\overline{\pi(\xi)} \overline{\pi(\eta)}}=$ $\overline{\pi(\xi \eta)}$ and $\lambda \cdot \overline{\pi(\xi)}=\overline{\pi(\lambda \xi)}$ are showed. By Lemma 2.2 (2) we have $\boldsymbol{J} \pi(\xi) \boldsymbol{J}=\pi^{\prime}(\xi)^{*}, \quad \xi \in \mathscr{D}$ and hence $\overline{\boldsymbol{J} \pi(\xi) \boldsymbol{J}}=\overline{\pi^{\prime}(\xi)^{*}}=\pi^{\prime}(\xi)^{*}$ by Lemma 2.1. On the other hand we can easily show $\overline{J \pi(\xi) J}=$ $\overline{J \pi(\xi)} J$. Therefore we have $\overline{J \pi(\xi)} J=\pi^{\prime}(\xi)^{*}$.

Problem. Does there exist an $E W^{*}$-algebra $\mathfrak{A}$ such that $\overline{\mathfrak{A}_{b}}=$ $\mathcal{U}_{0}\left(\mathscr{D}_{0}\right)$ and $\overline{\mathfrak{A}} \supset \overline{\pi(\mathscr{D})}$ ? 
In $\$ 3$ we show that there exist such $E W^{*}$-algebras. In particular, there exists an $E W^{*}$-algebra such that is minimal in such $E W^{*}$-algebras and we call it the left $E W^{*}$-algebra of $\mathscr{D}$.

We introduce examples of unbounded Hilbert algebras.

(1) $L^{\omega}[0,1]$. Let $L^{\omega}[0,1]$ be the set of all complex-valued measurable functions $f$ on $[0,1]$ such that $f \in L^{p}[0,1], p=1,2, \cdots$. By the whole collection of $L^{p}$-norms

$$
\|f\|_{p}=\left[\int_{0}^{1}|f(t)|^{p} d t\right]^{1 / p}, \quad \quad p=1,2, \cdots
$$

and by pointwise multiplication and involution $\left(f^{*}(t)=\overline{f(t)}, t \in[0,1]\right)$ the space $L^{\omega}[0,1]$ is a complete metrizable locally convex $*$-algebra with jointly continuous multiplication. $\mathrm{R}$. Arens [1] showed $L^{\omega}[0,1]$ is not a locally $m$-convex algebra. However, G. R. Allan [2] showed that $L^{\omega}[0,1]$ is a $G B^{*}$-algebra with $\left(L^{\omega}[0,1]\right)_{0}=L^{x}[0,1]$. We introduce the inner product into $L^{\omega}[0,1]$ by;

$$
(f \mid g)=\int_{0}^{1} f(t) \overline{g(t)} d t, \quad f, g \in L^{\omega}[0,1]
$$

Then $L^{\omega}[0,1]$ is regarded as a pure unbounded Hilbert algebra over $L^{\infty}[0,1]$.

(2) $L^{\omega}(-\infty, \infty)$. Let $L^{\omega}(-\infty, \infty)$ be the set of all complex-valued measurable functions $f$ on $(-\infty, \infty)$ such that $f \in L^{p}(-\infty, \infty)$ for every real number $p \geqq 1$. Under the following operations

$$
\begin{aligned}
& (f g)(x)=f(x) g(x), \quad(\lambda f)(x)=\lambda f(x), \\
& f^{*}(x)=\overline{f(x)}
\end{aligned}
$$

and inner product $(f \mid g)=\int_{-\infty}^{\infty} f(x) \overline{g(x)} d x$, we can show that $L^{\omega}(-\infty, \infty)$ is a pure unbounded Hilbert algebra.

(3) $L_{1}^{\omega}(G)$ and $L_{2}^{\omega}(G)$. Let $G$ be a unimodular locally compact group and let $d x$ be a Haar measure on $G$. Let $L^{p}(G)$ be the Banach space of measurable functions $f$ on $G$ for which the norm

$$
\begin{aligned}
& \|f\|_{p}=\left[\int_{G}|f(x)|^{p} d x\right]^{1 / p}, \quad 1 \leqq p<\infty \\
& \|f\|_{\infty}=\operatorname{ess} \sup |f(x)|
\end{aligned}
$$

is finite. We note 
$L(G)$; the space of complex-valued continuous functions with compact supports,

$$
\begin{aligned}
& L^{\omega}(G)=\bigcap_{1 \leqq p \leqq \infty} L^{p}(G), \quad L_{1}^{\omega}(G)=\bigcap_{1<p \leqq \infty} L^{p}(G), \\
& L_{2}^{\omega}(G)=\bigcap_{1<p \leqq 2} L^{p}(G) .
\end{aligned}
$$

Under the convolution $f * g$ as multiplication, involution $f^{*}\left(f^{*}(x)=\right.$ $\left.\overline{f\left(x^{-1}\right)}\right)$ and inner product $(f \mid g)=\int_{G} f(x) \overline{g(x)} d x$ on $L^{2}(G), L^{\omega}(G)$ is a Hilbert algebra and $L_{1}^{\omega}(G)$ and $L_{2}^{\omega}(G)$ are unbounded Hilbert algebras. In fact, suppose $f \in L^{p}(G)$ and $g \in L^{q}(G)(1 / p+1 / q \geqq 1)$. Then by Young's inequality $f * g$ exists and $\|f * g\|_{r} \leqq\|f\|_{p}\|g\|_{q}$ where $1 / r=1 / p+1 / q-1$. Furthermore, for each $f \in L^{p}(G)(1 \leqq p<\infty)$ we have $\left\|f^{*}\right\|_{p}=\|f\|_{p}$. Therefore we can easily show that $L^{\omega}(G), L_{1}^{\omega}(G)$ and $L_{2}^{\omega}(G)$ are * -algebras. Since $L(G) \subset L^{\omega}(G) \subset L^{1}(G) \cap L^{2}(G)$ and $L(G)$, $\mathrm{L}^{1}(\mathrm{G}) \cap \mathrm{L}^{2}(\mathrm{G})$ are Hilbert algebras, $L^{\omega}(G)$ is clearly a Hilbert algebra. We can easily show that $(f \mid g)=\left(g^{*} \mid f^{*}\right)$ and $(f * g \mid h)=$ $\left(g \mid f^{*} * h\right)$ for every $f, g, h \in L_{1}^{\omega}(G)$ (resp. $\left.L_{2}^{\omega}(G)\right)$. Furthermore we have

$$
\left.L^{\omega}(G) \subset\left(L_{1}^{\omega}(G)\right)_{0} \quad \text { (resp. } L_{2}^{\omega}(G)_{0}\right) \subset L^{2}(G),
$$

and so $\left(L_{1}^{\omega}(G)_{0}\right)^{2}$ (resp. $\left.\left(L_{2}^{\omega}(G)_{0}\right)^{2}\right)$ is dense in $L^{2}(G)$. Therefore $L_{1}^{\omega}(G)$ and $L_{2}^{\omega}(G)$ are unbounded Hilbert algebras.

Problem. Is an unbounded Hilbert algebra $L_{1}^{\omega}(G)\left(\right.$ or $\left.L_{2}^{\omega}(G)\right)$ pure?

If $G$ is a compact group, then $L^{2}(G)$ is an $H^{*}$-algebra, and so $L_{1}^{\omega}(G)$ and $L_{2}^{\omega}(G)$ are Hilbert algebras.

If $G=(-\infty, \infty)$, then

$$
L_{1 *}^{\omega}(-\infty, \infty)=\bigcap_{1<p \leqq \infty} L^{p}(-\infty, \infty)
$$

and

$$
L_{2^{*}}^{\omega}(-\infty, \infty)=\bigcap_{1<p \leqq 2} L^{p}(-\infty, \infty)
$$

are pure unbounded Hilbert algebras under the following operations and inner product 


$$
\begin{aligned}
& (f * g)(x)=\int_{-\infty}^{\infty} f(y) g(x-y) d y \\
& (\lambda f)(x)=\lambda f(x), \quad f^{*}(x)=\overline{f(-x)} \\
& (f \mid g)=\int_{-\infty}^{\infty} f(x) \overline{g(x)} d x
\end{aligned}
$$

In fact, we note

$$
\pi(f) g=f * g, \quad f, g \in L_{1^{*}}^{\omega}(-\infty, \infty)
$$

and

$$
\left(L_{1^{*}}^{\omega}(-\infty, \infty)\right)_{0}=\left\{f \in L_{1^{*}}^{\omega}(-\infty, \infty) ; \pi(f) \text { is continuous }\right\}
$$

We have only to show $\left(L_{1^{*}}^{\omega}(-\infty, \infty)\right)_{0} \neq L_{1^{*}}^{\omega}(-\infty, \infty)$. By the theory of Hilbert algebras we have

$$
\begin{aligned}
\left(L^{1}(-\infty, \infty) \cap L^{2}(-\infty, \infty)\right)_{b}= & \left\{f \in L^{2}(-\infty, \infty) ; \overline{\pi(f)}\right. \text { is a bounded } \\
& \text { linear operator on } \left.L^{2}(-\infty, \infty)\right\} \\
= & \left\{f \in L^{2}(-\infty, \infty) ; \hat{f} \in L^{\infty}(-\infty, \infty)\right\}
\end{aligned}
$$

where $\hat{f}$ denotes the Fourier transform of $f$. Clearly we have

$$
\left(L_{1^{*}}^{\omega}(-\infty, \infty)\right)_{0} \subset\left\{f \in L^{2}(-\infty, \infty) ; \hat{f} \in L^{\infty}(-\infty, \infty)\right\} .
$$

Putting

$$
f(x)=\left\{\begin{array}{cc}
0, & x<1 \\
1 / x, & x \geqq 1
\end{array}\right.
$$

we can show $f \in L_{1^{*}}^{\omega}(-\infty, \infty)$ and $\hat{f} \notin L^{\infty}(-\infty, \infty)$, and so $L_{1^{*}}^{\omega}(-\infty, \infty)_{0} \neq L_{1^{*}}^{\omega}(-\infty, \infty)$. Consequently $L_{1^{*}}^{\omega}(-\infty, \infty)$ is pure.

3. $L^{\omega}$-spaces with respect to noncommutative integration. Our starting point for the construction of $L^{\omega}$-space will be the algebras of operators measurable with respect to a von Neumann algebra as constructed by Segal in [14]. Let $\mathfrak{U}$ be a semifinite von Neumann algebra on a Hilbert space $\mathfrak{F}$ and let $\varphi$ be a faithful normal semifinite trace on $\mathfrak{U}^{+}$. Let $\mathfrak{U}_{p}$ and $\mathfrak{A}_{u}$, respectively, denote the set of all projections and that of unitary operators in $\mathfrak{U}$. 
Definition 3.1. A linear set $\mathfrak{D}$ in $\mathfrak{S}$ is said to be strongly dense (resp. $\varphi$-restrictedly strongly dense) provided

(a) $U^{\prime} \mathfrak{D} \subset \mathfrak{D}$ for every $U^{\prime} \in \mathfrak{A}_{u}^{\prime}$;

(b) there exists a sequence of projections $P_{n} \in \mathfrak{A}$ such that $P_{n} \mathfrak{S} \subset \mathfrak{D}$, $P_{n}^{\perp} \downarrow 0$ and $P_{n}^{\perp}$ is a finite projection (resp. $\varphi\left(P_{n}^{\perp}\right)<\infty$ ). An operator $T \eta \mathfrak{A}$ is called essentially measurable (resp. $\varphi$-restrictedly essentially measurable) if $T$ has a strongly dense (resp. $\varphi$-restrictedly strongly dense) domain and a closed extension. Moreover if $T$ is closed, $T$ is called measurable (resp. $\varphi$-restrictedly measurable).

LEMma 3.2. ([11] Lemma 1.1.) Let $T$ be a closed densely defined operator $\eta \mathfrak{A}$. Then;

(1) $T$ is measurable (resp. $\varphi$-restrictedly measurable) if and only if so is $|T|$.

(2) Let $T \geqq 0$ and let $T=\int_{0}^{\infty} \lambda d E(\lambda)$ be its spectral resolution. T is measurable (resp. $\varphi$-restrictedly measurable) if and only if $E(\lambda)^{\perp}(=I-$ $E(\lambda)$ ) is a finite projection (resp. $\varphi\left(E(\lambda)^{\perp}\right)<\infty$ ) for a positive $\lambda$.

We denote the set of all operators on $\mathfrak{F}$ measurable (resp. $\varphi$ restrictedly measurable) with respect to $\mathfrak{A}$ by $\mathfrak{M}(\mathfrak{U})(\operatorname{resp} . \mathfrak{M}(\varphi))$.

Proposition 3.3. ([7] Prop. 4.3.) The sets $\mathfrak{M}(\mathfrak{U})$ and $\mathfrak{M}(\varphi)$ form $E W^{*}$-algebras over $\mathfrak{A}$ under the operations of strong sum, strong product, adjoint and strong scalar multiplication.

Let $\mathfrak{M}_{\varphi}$ be the maximal ideal associated with $\varphi$, that is, the set of $A \in \mathfrak{U}$ with $\varphi(|A|)<\infty$. For every $T \in \mathfrak{M}(\mathfrak{U})^{+}$we put

$$
\mu(T)=\sup _{A \in \mathfrak{M}_{\varphi}, A \leqq T} \varphi(A) .
$$

Definition 3.4. A measurable operator $T \eta \mathfrak{A}$ is said to be $p$ th power integrable with respect to $\varphi$ if $\mu\left(|T|^{p}\right)<\infty$. Let $L^{p}(\varphi)$ $(1 \leqq p<\infty)$ stand for the set of $p$ th power integrable operators $\eta \mathfrak{A}$. The $L^{p}$-norm of $T \in L^{p}(\varphi)$ is defined as $\mu\left(|T|^{p}\right)^{1 / p}$ and designated by $\|T\|_{p}$. If $p=\infty$, we shall identify $\mathfrak{A}$ with $L^{\infty}(\varphi)$.

A measurable operator $T$ belongs to $L^{p}(\varphi)(1 \leqq p<\infty)$ if and only if $T$ is $\varphi$-restrictedly measurable and $-\int_{0}^{\infty} \lambda^{p} d \varphi\left(E(\lambda)^{\perp}\right)<\infty$, where $\int_{0}^{\infty} \lambda d E(\lambda)$ is the spectral resolution of $|T|$.

THEOREM 3.5. [11] (1) For $1 \leqq p<\infty L^{p}(\varphi)$ is a Banach space with norm $\|T\|_{p}$ and the following properties are satisfied. 
(a) $\|T\|_{p}=\left\|T^{*}\right\|_{p}=\left\|U \cdot T \cdot U^{*}\right\|_{p}$ for $T \in L^{p}(\varphi)$ and $U \in \mathfrak{A}_{u}$.

(b) For $S, T \in L^{p}(\varphi)$ such that $|T| \leqq|S|$ we have $\|T\|_{p} \leqq\|S\|_{p}$.

(c) For $A \in \mathfrak{A}$ and $T \in L^{p}(\varphi)$ we have $\|A \cdot T\|_{p} \leqq\|A\|\|T\|_{p}$.

(d) If $0 \leqq T_{1} \leqq T_{2} \leqq \cdots$ is a sequence of elements of $L^{p}(\varphi)$ such that $\left\{\left\|T_{n}\right\|_{p}\right\}$ is bounded, then there exists $T:=\sup T_{n}$ and $\lim _{n \rightarrow \infty}\left\|T-T_{n}\right\|_{p}=0$.

(2) Let $1 / p+1 / q=1$ where $1 \leqq p, q \leqq \infty$. Then

(a) $\mu(S \cdot T)=\mu(T \cdot S)$ for $S \in L^{p}(\varphi)$ and $T \in L^{q}(\varphi)$. If furthermore, $S, T \geqq 0$, then $\mu(S \cdot T) \geqq 0$; and conversely, if $\mu(S \cdot T) \geqq 0$ for every $T \geqq 0$, then $S \geqq 0$.

(b) $\quad\left|\mu\left(T_{1} \cdot T_{2} \cdots \cdot T_{n}\right)\right| \leqq \mu\left(\left|T_{1} \cdot T_{2} \cdots \cdot T_{n}\right|\right) \leqq\left\|T_{1}\right\|_{p_{1}}\left\|T_{2}\right\|_{p_{2}} \cdots$ $\left\|T_{n}\right\|_{p_{n}}$ for $T_{\imath} \in L^{p_{1}}(\varphi)$ with $\sum_{i=1}^{n} 1 / p_{\imath}=1, p_{i} \geqq 1(i=1,2, \cdots, n)$.

$$
\|S\|_{p}=\sup _{T \in L^{q}(\varphi),\|T\|_{q} \leqq 1}|\mu(S \cdot T)|
$$

for $S \in L^{p}(\varphi)$ where the sup is attained by some $T$ if $1 \leqq p<\infty$.

(d) $|\mu(S \cdot T)|^{2} \leqq \mu\left(\left|S^{*}\right| \cdot|T|\right) \mu\left(|S| \cdot\left|T^{*}\right|\right) \leqq \mu(|S \cdot T|) \mu(|T \cdot S|)$ for $S \in L^{p}(\varphi)$ and $T \in L^{q}(\varphi)$.

(3) Let $1 / p+1 / q=1 / r$ where $1 \leqq p, q, r \leqq \infty$.

(a) If $T \in L^{p}(\varphi)$ and $S \in L^{q}(\varphi)$, then $T \cdot S \in L^{r}(\varphi)$ and we have $\|T \cdot S\|_{r} \leqq\|T\|_{p}\|S\|_{q}$.

(b) Let $T$ be a $\varphi$-restrictedly measurable operator $\eta \mathfrak{A}$. If $T \cdot S \in$ $L^{r}(\varphi)$ for every $S \in L^{q}(\varphi)^{+}$, then $T \in L^{p}(\varphi)$.

Let $\mathscr{D}_{0}$ be a Hilbert algebra. Let $\mathscr{U}_{0}\left(\mathscr{D}_{0}\right)$ be the left von Neumann algebra of $\mathscr{D}_{0}$ and let $\varphi_{0}$ be the natural trace on $\mathscr{U}_{0}\left(\mathscr{D}_{0}\right)^{+}$. The completion $\mathfrak{S}$ of $\mathscr{D}_{0}$ is equivalent to an $H$-system [3]. Putting

$$
\left(\mathscr{D}_{0}\right)_{b}=\left\{x \in \mathfrak{S} ; \overline{\pi_{0}(x)} \text { is bounded }\right\}
$$

$\left(\mathscr{D}_{0}\right)_{b}$ is a maximal Hilbert algebra containing $\mathscr{D}_{0}$ and $\mathcal{U}_{0}\left(\mathscr{D}_{0}\right)\left(\mathscr{D}_{0}\right)_{b} \subset\left(\mathscr{D}_{0}\right)_{b}$. For every $x \in \mathfrak{S}_{\mathfrak{C}} \overline{\pi_{0}(x)}$ is $\varphi_{0}$-restrictedly measurable ([11] Lemma 2.3.). We can easily show that $L^{2}\left(\varphi_{0}\right)=\left\{\pi_{0}(x) ; x \in \mathfrak{S}_{\mathcal{C}}\right\}$ and $L^{2}\left(\varphi_{0}\right)$ is a Hilbert space isometric with $\mathfrak{S}$. Moreover we remark that $L^{2}\left(\varphi_{0}\right)$ is an $H$-system isomorphic with $\mathfrak{S}$ by the map. $x \rightarrow \overline{\pi_{0}(x)}$. This follows from the facts that (1) if $x y$ is defined and equals $z$, then $\pi_{0}(x) \cdot \overline{\pi_{0}(y)}=\overline{\pi_{0}(x y)}$ and (2) if $\overline{\pi_{0}(x)} \cdot \overline{\pi_{0}(y)}$ equals $\overline{\pi_{0}(z)}$, then $x y$ is defined and equals $z$. We have

$$
L^{1}\left(\varphi_{0}\right)=\left\{\sum_{i=1}^{m} \overline{\pi_{0}\left(x_{i}\right)} \cdot \overline{\pi_{0}\left(y_{i}\right)} ; x_{i}, y_{t} \in \mathfrak{S}\right\}
$$

and the integral $\mu(T)$ of $T=\sum_{i=1}^{m} \overline{\pi_{0}\left(x_{i}\right)} \cdot \overline{\pi_{0}\left(y_{\imath}\right)}$ equals $\sum_{i=1}^{m}\left(y_{\imath} \mid x_{\imath}^{*}\right)$. 
Definition 3.5. We define the $L^{\omega}$-spaces with respect to the natural trace $\varphi_{0}$ as follows;

$$
\begin{aligned}
& L^{\omega}\left(\varphi_{0}\right)=\bigcap_{1 \leqq p<\infty} L^{p}\left(\varphi_{0}\right), \\
& L_{2}^{\omega}\left(\varphi_{0}\right)=\bigcap_{2 \leqq p<\infty} L^{p}\left(\varphi_{0}\right) .
\end{aligned}
$$

Similarly we define the $L^{\omega}$-spaces with respect to the Hilbert algebra $\mathscr{D}_{0}$ as follows;

$$
\begin{aligned}
& L^{\omega}\left(\mathscr{D}_{0}\right)=\left\{x \in \mathfrak{H} ; \overline{\pi_{0}(x)} \in L^{\omega}\left(\varphi_{0}\right)\right\}, \\
& L_{2}^{\omega}\left(\mathscr{D}_{0}\right)=\left\{x \in \mathfrak{S} ; \overline{\pi_{0}(x)} \in L_{2}^{\omega}\left(\varphi_{0}\right)\right\} .
\end{aligned}
$$

Proposition 3.6. The space $L^{\omega}\left(\mathscr{D}_{0}\right)$ (resp. $\left.L_{2}^{\omega}\left(\mathscr{D}_{0}\right)\right)$ is an unbounded Hilbert algebra containing $\left(\mathscr{D}_{0}\right)_{b}^{2}\left(\right.$ resp. $\left.\left(\mathscr{D}_{0}\right)_{b}\right)$.

Proof. For $1 \leqq p<\infty$ and $S, T \in L^{\omega}\left(\varphi_{0}\right)$

$$
\|S \cdot T\|_{p} \leqq\|S\|_{2 p}\|T\|_{2 p}
$$

and hence $S \cdot T \in L^{\omega}\left(\varphi_{0}\right)$. Therefore, for each $x$ and $y$ in $L^{\omega}\left(\mathscr{D}_{0}\right) x y$ is defined and equals $\overline{\pi_{0}(x) y}$. Furthermore for each $T \in L^{p}\left(\varphi_{0}\right)$ $(1 \leqq p<\infty) \quad\|T\|_{p}=\left\|T^{*}\right\|_{p}$ and hence $x^{*} \in L^{\omega}\left(\mathscr{D}_{0}\right)$ for every $x \in$ $L^{\omega}\left(\mathscr{D}_{0}\right)$. Consequently $L^{\omega}\left(\mathscr{D}_{0}\right)$ is a $*$-algebra. We can easily show $L^{\omega}\left(\mathscr{D}_{0}\right) \supset\left(\mathscr{D}_{0}\right)_{b}^{2}$, and so $L^{\omega}\left(\mathscr{D}_{0}\right)$ is a pre-Hilbert space and its completion is $L^{2}\left(\mathscr{D}_{0}\right)=\mathfrak{S}_{\mathcal{C}}$. For every $x, y$ and $z$ in $L^{\omega}\left(\mathscr{D}_{0}\right)$ we have

$$
(x \mid y)=\left(y^{*} \mid x^{*}\right)
$$

and

$$
(x y \mid z)=\left(\overline{\pi_{0}(x)} y \mid z\right)=\left(y \mid \pi_{0}(x)^{*} z\right)=\left(y \mid \overline{\pi_{0}\left(x^{*}\right)} z\right)=\left(y \mid x^{*} z\right) .
$$

Consequently $L^{\omega}\left(\mathscr{D}_{0}\right)$ is an unbounded Hilbert algebra. Similarly we can show that $L_{2}^{\omega}\left(\mathscr{D}_{0}\right)$ is an unbounded Hilbert algebra containing $\left(\mathscr{D}_{0}\right)_{b}$.

Proposition 3.7. The space $L^{\omega}\left(\varphi_{0}\right)$ (resp. $\left.L_{2}^{\omega}\left(\varphi_{0}\right)\right)$ is an unbounded Hilbert algebra containing $\overline{\pi_{0}\left(\left(\mathscr{D}_{0}\right)_{b}\right)^{2}}$ (resp. $\pi_{0}\left(\left(\mathscr{D}_{0}\right)_{b}\right)$ ) under the strong sum, strong product, adjoint, strong scalar multiplication and inner product on $L^{2}\left(\varphi_{0}\right)$. 
Proof. We can easily show that the map $x \in \mathfrak{H} \rightarrow \overline{\pi_{0}(x)} \in L^{2}\left(\varphi_{0}\right)$ is an isometric isomorphism of $L^{\omega}\left(\mathscr{D}_{0}\right)$ onto $L^{\omega}\left(\varphi_{0}\right)$. By Proposition 3.6. $L^{\omega}\left(\varphi_{0}\right)$ is an unbounded Hilbert algebra.

Problem. Is $L^{\omega}\left(\mathscr{D}_{0}\right)$ a pure unbounded Hilbert algebra? Does there exist a pure unbounded Hilbert algebra containing $\mathscr{D}_{0}$ ?

Proposition 3.8. The following conditions are equivalent.

(1) There exists a pure unbounded Hilbert algebra $\mathscr{D}$ containing $\mathscr{D}_{0}$.

(2) $L_{2}^{\omega}\left(\mathscr{D}_{0}\right)$ is a pure unbounded Hilbert algebra.

(3) $L^{\omega}\left(\mathscr{D}_{0}\right)$ is a pure unbounded Hilbert algebra.

(4) There exists a positive element $x$ in $\mathfrak{S}$ (i.e., $\overline{\pi_{0}(x)} \geqq 0$ ) such that $x \notin\left(\mathscr{D}_{0}\right)_{b}$ and $x^{n} \in \mathfrak{F}, n=1,2, \cdots$.

Proof. $\quad(1) \Rightarrow(4)$; There exists an element $\xi \in \mathscr{D}$ such that $\overline{\pi(\xi)}$ is an unbounded operator on $\mathfrak{S}$. Clearly $\xi^{*} \xi \notin\left(\mathscr{D}_{0}\right)_{b}$ and $\left(\xi^{*} \xi\right)^{n} \in \mathscr{D} \subset \mathfrak{S}$, $n=1,2, \cdots$.

(4) $\Rightarrow(3)$; Let $y=x^{2}$. Then we can easily show that $y \notin\left(\mathscr{D}_{0}\right)_{b}$ and for each positive integer $n \overline{\pi_{0}(y)} \in L^{n}\left(\varphi_{0}\right)$. Let $\overline{\pi_{0}(y)}=\int_{0}^{\infty} \lambda d E(\lambda)$ be the spectral resolution. For each $p$ with $1 \leqq p<\infty$ there is a positive integer $n$ such that $n \leqq p<n+1$. Then we have

$$
\begin{aligned}
-\int_{0}^{\infty} \lambda^{p} d \varphi_{0}\left(E(\lambda)^{\perp}\right) & \leqq-\int_{0}^{1} \lambda^{n} d \varphi_{0}\left(E(\lambda)^{\perp}\right)-\int_{1}^{\infty} \lambda^{n+1} d \varphi_{0}\left(E(\lambda)^{\perp}\right) \\
& \leqq-\int_{0}^{\infty} \lambda^{n} d \varphi_{0}\left(E(\lambda)^{\perp}\right)-\int_{0}^{\infty} \lambda^{n+1} d \varphi_{0}\left(E(\lambda)^{\perp}\right) \\
& <\infty
\end{aligned}
$$

Therefore $\overline{\pi_{0}(y)} \in L^{p}\left(\varphi_{0}\right)$, i.e., $y \in L^{p}\left(\mathscr{D}_{0}\right)$ for every $1 \leqq p<\infty$, and so $y \in L^{\omega}\left(\mathscr{D}_{0}\right)$ and $\pi_{0}(y)$ is unbounded. Consequently $L^{\omega}\left(\mathscr{D}_{0}\right)$ is a pure unbounded Hilbert algebra.

(3) $\Rightarrow$ (2); Since $L^{\omega}\left(\mathscr{D}_{0}\right) \subset L_{2}^{\omega}\left(\mathscr{D}_{0}\right)$, the assertion (3) $\Rightarrow$ (2) is obvious.

$(2) \Rightarrow(1) ; L_{2}^{\omega}\left(\mathscr{D}_{0}\right)$ is a pure unbounded Hilbert algebra containing $\mathscr{D}_{0}$.

THEOREM 3.9. Let $\mathscr{D}$ be a pure unbounded Hilbert algebra over $\mathscr{D}_{0}$. Then $\mathscr{D}^{2}$ (resp. $\left.\mathscr{D}\right)$ is a*-subalgebra of the pure unbounded Hilbert algebra $L^{\omega}\left(\mathscr{D}_{0}\right)$ (resp. $L_{2}^{\omega}\left(\mathscr{D}_{0}\right)$ ). In particular, $L_{2}^{\omega}\left(\mathscr{D}_{0}\right)$ is maximal in pure unbounded Hilbert algebras containing $\mathscr{D}_{0}$.

Proof. By Proposition $3.8 L^{\omega}\left(\mathscr{D}_{0}\right)$ and $L_{2}^{\omega}\left(\mathscr{D}_{0}\right)$ are pure unbounded Hilbert algebras. In the same way as the proof $(4) \Rightarrow(3)$ of Proposition 3.8 we can easily show $L^{\omega}\left(\mathscr{D}_{0}\right) \supset \mathscr{D}^{2}$ and $L_{2}^{\omega}\left(\mathscr{D}_{0}\right) \supset \mathscr{D}$. 
Problem. Let $\mathscr{D}$ be a pure unbounded Hilbert algebra over $\mathscr{D}_{0}$. Does there exist an $E W^{*}$-algebra $\mathfrak{U}$ such that $\overline{\mathfrak{A}_{b}}=\mathscr{U}_{0}\left(\mathscr{D}_{0}\right)$ and $\overline{\mathfrak{A}} \supset \overline{\pi(\mathscr{D})}$ ?

Let $\mathscr{D}$ be a pure unbounded Hilbert algebra over $\mathscr{D}_{0}$. By Proposition $3.8 L_{2}^{\omega}\left(\mathscr{D}_{0}\right)$ is a pure unbounded Hilbert algebra such that

$$
\mathscr{D}_{0} \subset \mathscr{D} \subset L_{2}^{\omega}\left(\mathscr{D}_{0}\right) \subset \mathfrak{S}, \quad \text { and } \quad L^{\infty}\left(\varphi_{0}\right) L_{2}^{\omega}\left(\mathscr{D}_{0}\right) \subset L_{2}^{\omega}\left(\mathscr{D}_{0}\right) \text {. }
$$

Let $\pi$ (resp. $\pi_{2}^{\omega}$ ) be the left regular representation of $\mathscr{D}$ (resp. $\left.L_{2}^{\omega}\left(\mathscr{D}_{0}\right)\right)$. By Lemma 2.1 we have $\overline{\pi_{2}^{\omega}(\mathscr{D})}=\overline{\pi(\mathscr{D})}=\overline{\pi_{0}(\mathscr{D})}$.

Then $\pi_{2}^{\omega}(\mathscr{D})$ is a \# -algebra on $L_{2}^{\omega}\left(\mathscr{D}_{0}\right)$ under $\pi_{2}^{\omega}(\xi)^{\#}=\pi_{2}^{\omega}\left(\xi^{*}\right)$ and $L^{\infty}\left(\varphi_{0}\right) / L_{2}^{\omega}\left(\mathscr{D}_{0}\right):=\left\{T / L_{2}^{\omega}\left(\mathscr{D}_{0}\right) ; \quad T \in L^{\infty}\left(\varphi_{0}\right)\right\}$ is a \#-algebra on $L_{2}^{\omega}\left(\mathscr{D}_{0}\right)$ under $\left(T / L_{2}^{\omega}\left(\mathscr{D}_{0}\right)\right)^{*}=T^{*} / L_{2}^{\omega}\left(\mathscr{D}_{0}\right)$, where $T / L_{2}^{\omega}\left(\mathscr{D}_{0}\right)$ is the restriction of $T$ onto $L_{2}^{\omega}\left(\mathscr{D}_{0}\right)$.

Notation. We denote by $\mathcal{U}(\mathscr{D})$ a \# -algebra on $L_{2}^{\omega}\left(\mathscr{D}_{0}\right)$ generated by $\pi_{2}^{\omega}(\mathscr{D})$ and $L^{\infty}\left(\varphi_{0}\right) / L_{2}^{\omega}\left(\mathscr{D}_{0}\right)$.

THEOREM 3.10. Let $\mathscr{D}$ be a pure unbounded Hilbert algebra over $\mathscr{D}_{0}$. Then $\mathcal{U}(\mathscr{D})$ and $\mathcal{U}\left(L_{2}^{\omega}\left(\mathscr{D}_{0}\right)\right)$ are $E W^{*}$-algebras on $L_{2}^{\omega}\left(\mathscr{D}_{0}\right)$ such that $\overline{U(\mathscr{D})_{b}}=\overline{\mathcal{U}\left(L_{2}^{\omega}\left(\mathscr{D}_{0}\right)\right)_{b}}=\mathcal{U}_{0}\left(\mathscr{D}_{0}\right)$ and $\overline{U\left(L_{2}^{\omega}\left(\mathscr{D}_{0}\right)\right)} \supset \overline{\mathcal{U}(\mathscr{D})} \supset \overline{\pi(\mathscr{D})}$.

Definition 3.11. Let $\mathscr{D}$ be a pure unbounded Hilbert algebra over $\mathscr{D}_{0}$. $\quad \mathcal{U}(\mathscr{D})$ is called the left $E W^{\#}$-algebra of $\mathscr{D}$.

4. Traces on $E W^{*}$-algebras. Let $\mathfrak{A}$ be an $E W^{*}$-algebra and let $\varphi$ be a trace on $\mathfrak{A}^{+}$. We note

$$
\mathfrak{N}_{\varphi}=\left\{T \in \mathfrak{A} ; \varphi\left(T^{\#} T\right)<\infty\right\}
$$

and let $\mathfrak{M}_{\varphi}$ be a linear combination of $\left\{S T^{\#} ; S, T \in \mathfrak{N}_{\varphi}\right\}$. Then, clearly, $\mathfrak{N}_{\varphi}$ (resp. $\mathfrak{M}_{\varphi}$ ) is a \# -subspace of $\mathfrak{A}$ satisfying $\mathfrak{U}_{b} \mathfrak{N}_{\varphi} \subset \mathfrak{N}_{\varphi}$ and $\mathfrak{N}_{\varphi} \mathfrak{U}_{b} \subset$ $\mathfrak{N}_{\varphi}$ (resp. $\mathfrak{H}_{b} \mathfrak{M}_{\varphi} \subset \mathfrak{M}_{\varphi}$ and $\mathfrak{M}_{\varphi} \mathfrak{A}_{b} \subset \mathfrak{M}_{\varphi}$ ). We can easily show that the positive part $\mathfrak{M}_{\varphi}^{+}$of $\mathfrak{M}_{\varphi}$ equals $\left\{T \in \mathfrak{U}^{+} ; \varphi(T)<\infty\right\}$ and $\mathfrak{M}_{\varphi}$ is a linear combination of $\mathfrak{M}_{\varphi}^{+}$. We define $\dot{\varphi}$ by;

$$
\begin{gathered}
\dot{\varphi}(S)=\lambda_{1} \varphi\left(S_{1}\right)+\cdots+\lambda_{n} \varphi\left(S_{n}\right), \quad S=\lambda_{1} S_{1}+\cdots+\lambda_{n} S_{n}, \\
\lambda_{i} \in \mathcal{S}, \quad S_{i} \in \mathfrak{M}_{\varphi}^{+} .
\end{gathered}
$$

Then it is not difficult to show that $\dot{\varphi}$ is a well-defined linear form on $\mathfrak{M}_{\varphi}$ and it satisfies 
(1) $\dot{\varphi}(S)=\varphi(S), \quad S \in \mathfrak{M}_{\varphi}^{+}$;

(2) $\dot{\varphi}\left(S^{*} T\right)=\dot{\varphi}\left(T S^{*}\right), \quad S, T \in \mathfrak{N}_{\varphi}$;

(3) $\dot{\varphi}(S T)=\dot{\varphi}(T S), \quad S \in \mathfrak{M}_{\varphi}, \quad T \in \mathfrak{H}_{b}$.

We note

$$
\bar{\varphi}(\bar{T})=\varphi(T), \quad T \in \mathfrak{A}_{b}^{+} .
$$

Then $\bar{\varphi}$ is a trace on $\overline{\mathfrak{A}}_{b}^{+}$and we have

$$
\overline{\left(\mathfrak{N}_{\varphi}\right)_{b}}=\mathfrak{N}_{\bar{\varphi}} \quad \text { and } \quad \overline{\left(\mathfrak{M}_{\varphi}\right)_{b}}=\mathfrak{M}_{\bar{\varphi}} .
$$

Definition 4.1. Let $\mathfrak{A}$ be an $E W^{*}$-algebra and let $\varphi$ be a trace on $\mathfrak{H}^{+}$. If every $\bar{A} \in \overline{\mathfrak{A}}$ is $\bar{\varphi}$-restrictedly measurable, then $\mathfrak{U}$ is called $\varphi$-measurable.

Let $\mathscr{D}$ be a pure unbounded Hilbert algebra over $\mathscr{D}_{0}$ and let $\mathscr{S}_{\mathfrak{L}}$ be the completion of $\mathscr{D}$. Let $\mathscr{E}$ be a pure unbounded Hilbert algebra over $\left(\mathscr{D}_{0}\right)_{b}$ containing $\mathscr{D}$. Let $\mathfrak{A}$ be a $\varphi_{0}$-measurable (merely measurable) $E W^{*}$ algebra on $\mathscr{E}$ such that $\overline{\mathfrak{A}}_{b}=\mathscr{U}_{o}\left(\mathscr{D}_{0}\right)$ and $\overline{\mathfrak{A}} \supset \overline{\pi(\mathscr{D})}(\mathcal{U}(\mathscr{D})$ and $\mathcal{U}\left(L_{2}^{\omega}\left(\mathscr{D}_{0}\right)\right)$ are examples of such $E W^{*}$-algebras $)$, where $\varphi_{0}$. is the natural trace on $\mathscr{U}_{0}\left(\mathscr{D}_{0}\right)^{+}$.

Notation. For each $S \in \mathfrak{A}^{+}$we define $\varphi$ as follows;

$$
\varphi(S)=\left\{\begin{array}{cl}
(x \mid x), & \text { if } \overline{S^{1 / 2}}=\overline{\pi_{0}(x)}, x \in L_{2}^{\omega}\left(\mathscr{D}_{0}\right) \\
\infty, & \text { if otherwise. }
\end{array}\right.
$$

THEOREM 4.2. (1) $\varphi$ is a faithful normal semifinite trace on $\mathfrak{A}^{+}$.

(2) We have

$$
\overline{\mathfrak{R}}_{\varphi}=\overline{\mathfrak{A}} \cap L_{2}^{\omega}\left(\varphi_{0}\right) \text { and } \overline{\mathfrak{M}}_{\varphi}=\overline{\mathfrak{A}} \cap L^{\omega}\left(\varphi_{0}\right)
$$

(3) Putting

$\mathfrak{N}\left(\mathscr{D}_{0}\right)=\left\{x \in \mathfrak{H} ; \overline{\pi_{0}(x)} \in \overline{\mathfrak{N}_{\varphi}}\right\}$ and $\mathfrak{M}\left(\mathscr{D}_{0}\right)=\left\{x \in \mathfrak{S} ; \overline{\pi_{0}(x)} \in \overline{\mathfrak{M}_{\varphi}}\right\}$,

$\mathfrak{N}\left(\mathscr{D}_{11}\right)$ (resp. $\left.\mathfrak{M}\left(\mathscr{D}_{0}\right)\right)$ is a pure unbounded Hilbert algebra over $\left(\mathscr{D}_{0}\right)_{b}$ (resp. $\left.\left(\mathscr{D}_{1}\right)_{b}^{2}\right)$ containing $\mathscr{D}$ (resp. $\left.\mathscr{D}^{2}\right)$.

(4) $\bar{\varphi}$ equals the natural trace $\varphi_{0}$ on $U_{0}\left(\mathscr{D}_{0}\right)^{+}$.

(5) Let $\mu$ be the integral on $L^{1}\left(\varphi_{0}\right)$. Then

$$
\dot{\varphi}(T)=\mu(\bar{T}), \quad T \in \mathfrak{M}_{\varphi} .
$$


In particular, for every $x, y \in \mathfrak{N}\left(\mathscr{D}_{0}\right)$

$$
\dot{\varphi}\left(\overline{\pi_{0}(y)^{*}} \cdot \overline{\pi_{0}(x)}\right)=(x \mid y) .
$$

(6) $\mathfrak{A}\left(\mathfrak{N}_{\varphi}\right)_{b} \subset \mathfrak{N}_{\varphi}$ and $\mathfrak{A}\left(\mathfrak{M}_{\varphi}\right)_{b} \subset \mathfrak{M}_{\varphi}$.

(7) Every element $T$ of $\mathfrak{A}$ is represented by

$$
T=T_{0}+T_{1}, \quad T_{0} \in \mathfrak{U}_{b}, \quad T_{1} \in \mathfrak{M}_{\varphi} .
$$

(8) If $T \in \mathfrak{N}$, then we have $\bar{T}=\overline{\left(T / \mathscr{D}_{0}\right)}$.

Proof. (2); Let $T \in \mathfrak{N}_{\varphi}$ and let $T=U|T|$ be the polar decomposition of $T$. Since $\varphi\left(T^{*} T\right)=\varphi\left(|T|^{2}\right)<\infty, \overline{|T|}=\overline{\pi_{0}(x)}, x \in L_{2}^{\omega}\left(\mathscr{D}_{0}\right)$, and so $\overline{|T|} \in L_{2}^{\omega}\left(\varphi_{0}\right)$ and hence $\bar{T}=\bar{U} \cdot|T| \in L_{2}^{\omega}\left(\varphi_{0}\right) \cap \overline{\mathfrak{A}}$. The converse is obvious. Moreover we get

$$
\overline{\mathfrak{M}_{\varphi}}=\overline{\mathfrak{N}}_{\varphi}^{2}=\left(\overline{\mathfrak{A}} \cap L_{2}^{\omega}\left(\varphi_{0}\right)\right)^{2}=\overline{\mathfrak{A}} \cap L^{\omega}\left(\varphi_{0}\right) .
$$

(3); By (2) we can easily show (3).

(4); Let $T \in \mathfrak{I}_{b}^{+}$. Since $\overline{\mathfrak{I}_{b}} \cap L_{2}^{\omega}\left(\varphi_{0}\right)=\overline{\pi_{0}\left(\left(\mathscr{D}_{0}\right)_{b}\right)}$,

$$
\begin{aligned}
\bar{\varphi}(\bar{T})=\varphi(T) & =\left\{\begin{array}{cl}
(x \mid x), & \text { if } \overline{T^{1 / 2}}=\overline{\pi_{0}(x)}, x \in L_{2}^{\omega}\left(\mathscr{D}_{0}\right) ; \\
\infty, & \text { if otherwise }
\end{array}\right. \\
& =\left\{\begin{array}{cl}
(x \mid x), & \text { if } \overline{T^{1 / 2}}=\overline{\pi_{0}(x)}, x \in\left(\mathscr{D}_{0}\right)_{b} ; \\
\infty, & \text { if otherwise }
\end{array}\right. \\
& =\varphi_{0}(\bar{T}) .
\end{aligned}
$$

(5); Let $T \in \mathfrak{M}_{\varphi}^{+}$. By (2) there exists an element $x$ of $L_{2}^{\omega}\left(\mathscr{D}_{0}\right)$ such that $\overline{T^{1 / 2}}=\overline{\pi_{0}(x)}$. Then we have $\varphi(T)=(x \mid x)=\mu(\bar{T})$, and so $\dot{\varphi}(T)=$ $\mu(\bar{T}), T \in \mathfrak{M}_{\varphi}$.

(6); Let $\pi$ be the left regular representation of $\mathscr{E}$. We can easily show that

$$
T \pi(\xi)=\pi(T \xi), \quad T \in \mathfrak{A}, \quad \xi \in\left(\mathscr{D}_{0}\right)_{b} \subset \mathscr{E} .
$$

Therefore $\pi(T \xi)=T \pi(\xi) \in \mathfrak{A}$ and $\overline{\pi(T \xi)}=\overline{\pi_{0}(T \xi)}, \quad T \xi \in \mathscr{E} \subset L_{2}^{\omega}\left(\mathscr{D}_{0}\right)$, and so $T \pi(\xi) \in \mathfrak{N}_{\varphi}$.

(7); Let $T \in \mathfrak{U}$ and let $T=U|T|$ be the polar decomposition of T. Let $\overline{|T|}=\int_{0}^{\infty} \lambda d \overline{E_{T}(\lambda)}$ be the spectral resolution of $\overline{|T|}$. Since $\overline{|T|}$ is 
a $\varphi_{0}$-restrictedly measurable operator, $\overline{E_{T}\left(\lambda_{0}\right)^{\perp}} \in \overline{\left(\mathfrak{M}_{\varphi}\right)_{b}}$ for some $\lambda_{0}>$ 0. By (6) $\mathfrak{H}\left(\mathfrak{M}_{\varphi}\right)_{b} \subset \mathfrak{M}_{\varphi}$, and so putting

$$
T_{1}=T E_{T}\left(\lambda_{0}\right)^{\perp}=U|T| E_{T}\left(\lambda_{0}\right)^{\perp} \text { and } T_{0}=T E_{T}\left(\lambda_{0}\right),
$$

$T_{0} \in \mathfrak{A}_{b}, T_{1} \in \mathfrak{M}_{\varphi}$ and $T=T_{0}+T_{1}$.

(8); Let $T \in \mathfrak{A}$. By (7) we have

$$
\begin{aligned}
\bar{T} & =\bar{T}_{0}+\bar{T}_{1}, \quad T_{0} \in \mathfrak{U}_{b}, \quad T_{1} \in \mathfrak{M}_{\varphi} \\
& =\overline{T_{0}}+\overline{\pi_{0}(x)}, \quad x \in L^{\omega}\left(\mathscr{D}_{0}\right) \\
& =\overline{\left(T_{0} / \mathscr{D}_{0}\right)}+\overline{\pi_{0}(x)}=\overline{T_{0} / \mathscr{D}_{0}+\pi_{0}(x)}=\overline{T / \mathscr{D}_{0}} .
\end{aligned}
$$

(1); We shall show that $\varphi$ is a trace on $\mathfrak{A}^{+}$, i.e.,

(a) $\varphi(S+T)=\varphi(S)+\varphi(T), S, T \in \mathfrak{A}^{+}$;

(b) $\varphi(\lambda S)=\lambda \varphi(S), \lambda \geqq 0, S \in \mathfrak{U}^{+}$;

(c) $\varphi\left(S^{*} S\right)=\varphi\left(S S^{*}\right), S \in \mathfrak{A}$.

(a); Let $S, T \in \mathfrak{U}^{+}$. Suppose $\varphi(S+T)<\infty$. Since $\bar{S}$ (or $\left.\bar{T}\right) \leqq$ $\bar{S}+\bar{T}$ and $\bar{S}+\bar{T} \in \overline{\mathfrak{M}}_{\varphi}^{+}, \bar{S}$ and $\bar{T}$ in $\overline{\mathfrak{M}}_{\varphi}^{+}$, and so $\varphi(S)=\mu(\bar{S})<\infty$ and $\varphi(T)=\mu(\bar{T})<\infty$ by (5). Suppose $\varphi(S)<\infty$ and $\varphi(T)<\infty$. Since $\bar{S}$ and $\bar{T}$ in $L^{1}\left(\varphi_{0}\right)^{+}$, by Theorem 3.5. we have $\bar{S}+\bar{T} \in L^{1}\left(\varphi_{0}\right)^{+}$and

$$
\varphi(S)+\varphi(T)=\mu(\bar{S})+\mu(\bar{T})=\mu(\bar{S}+\bar{T})=\mu(\overline{S+T})=\varphi(S+T) .
$$

(b); clear.

(c); Let $S \in \mathfrak{A}$. Suppose $\varphi\left(S^{\#} \underline{S}\right)<\infty$. Let $S=U|S|$ be the polar decomposition of $S$. Then $|S|=\overline{\pi_{0}(x)}, x \in L_{2}^{\omega}\left(\mathscr{D}_{0}\right)$ and $\overline{\left|S^{*}\right|}=\left|S^{*}\right|=$ $\overline{\pi_{0}\left(x^{*}\right)}$, and so we get

$$
\varphi\left(S^{*} S\right)=(x \mid x)=\left(x^{*} \mid x^{*}\right)=\varphi\left(S S^{*}\right) .
$$

Consequently $\varphi$ is a trace on $\mathfrak{Q}^{+}$. Since $\bar{\varphi}=\varphi_{0}$ by (4), $\bar{\varphi}$ is a faithful normal semifinite trace on $\overline{\mathfrak{A}}_{b}^{+}$. We can easily show that $\varphi$ is faithful. We shall show that $\varphi$ is normal. Let $T_{\alpha} \uparrow T, T_{\alpha}, T \in \mathfrak{A}^{+}$. Suppose $\varphi(T)<\infty$. Then there exist $\left\{x_{\alpha}\right\} \subset L_{2}^{\omega}\left(\mathscr{D}_{0}\right)$ and $x \in L_{2}^{\omega}\left(\mathscr{D}_{0}\right)$ such that $\overline{T_{\alpha}^{1 / 2}}=\pi_{0}\left(x_{\alpha}\right)$ and $\overline{T^{1 / 2}}=\overline{\pi_{0}(x)}$. We can easily show that $\varphi\left(T_{\alpha}\right)=$ $\left\|x_{\alpha}\right\|^{2} \uparrow \varphi(T)=\|x\|^{2}$. Suppose $\varphi(T)=\infty$ and $\sup _{\alpha} \varphi\left(T_{\alpha}\right)<\infty$. There exists a net $\left\{x_{\alpha}\right\}$ of $L_{2}^{\omega}\left(\mathscr{D}_{0}\right)$ such that $\overline{T_{\alpha}^{1 / 2}}=\overline{\pi_{0}\left(x_{\alpha}\right)}$. Let $\bar{T}=\int_{0}^{\infty} \lambda d \overline{E_{T}(\lambda)}$ be the spectral resolution of $\bar{T}$. Since $\bar{T}$ is $\varphi_{0}$-restrictedly measurable, $\overline{E_{T}\left(\lambda_{0}\right)^{\perp}} \in \overline{\left(\mathcal{M}_{\varphi}\right)_{b}}{ }^{+}$for some $\lambda_{0}>0$, and so by (5) we get 


$$
T E_{T}\left(\lambda_{0}\right)^{\perp} \in \mathfrak{M}_{\varphi}^{+} \quad \text { and } \quad \bar{T}=\int_{0}^{\lambda_{0}} \lambda d \overline{E_{T}(\lambda)}+\bar{T} \overline{E_{T}\left(\lambda_{0}\right)^{\perp}} .
$$

From $\varphi(T)=\infty$, we have $\bar{\varphi}\left(\int_{0}^{\lambda_{0}} \lambda d \overline{E_{T}(\lambda)}\right)=\infty$. Since $T_{\alpha} \uparrow T$, we get $E_{T}\left(\lambda_{0}\right) T_{\alpha} E_{T}\left(\lambda_{0}\right) \in \mathfrak{A}_{b}$ and

$$
E_{T}\left(\lambda_{0}\right) T_{\alpha} E_{T}\left(\lambda_{0}\right) \uparrow E_{T}\left(\lambda_{0}\right) T E_{T}\left(\lambda_{0}\right)=\int_{0}^{\lambda_{0}} \lambda d E_{T}(\lambda) .
$$

Then we can show that

$$
\overline{E_{T}\left(\lambda_{0}\right) T_{\alpha} E_{T}\left(\lambda_{0}\right)} \uparrow \int_{0}^{\lambda_{0}} \lambda d \overline{E_{T}(\lambda)},
$$

and so by the normality of $\bar{\varphi}$

$$
\bar{\varphi}\left(\overline{E_{T}\left(\lambda_{0}\right) T_{\alpha} E_{T}\left(\lambda_{0}\right)}\right) \uparrow \bar{\varphi}\left(\int_{0}^{\lambda_{o}} \lambda d \overline{E_{T}(\lambda)}\right)=\infty .
$$

On the other hand we have

$$
\begin{aligned}
\bar{\varphi}\left(\int_{0}^{\lambda_{0}} \lambda d \overline{E_{T}(\lambda)}\right) & =\sup _{\alpha} \bar{\varphi}\left(\overline{E_{T}\left(\lambda_{0}\right) T_{\alpha} E_{T}\left(\lambda_{0}\right)}\right) \\
& \left.=\sup _{\alpha} \bar{\varphi} \overline{\left(\overline{E_{T}\left(\lambda_{0}\right)}\right.} \cdot \overline{\pi_{0}\left(x_{\alpha}\right)^{2}} \cdot \overline{E_{T}\left(\lambda_{0}\right)}\right) \\
& \left.=\sup _{\alpha} \bar{\varphi} \overline{\left(\pi_{0}\left(\overline{E_{T}\left(\lambda_{0}\right)} x_{\alpha}\right)\right.} \cdot \pi_{0}\left(\overline{E_{T}\left(\lambda_{0}\right)} x_{\alpha}^{*}\right)^{*}\right) \\
& =\sup _{\alpha}\left(\overline{E_{T}\left(\lambda_{0}\right)} x_{\alpha} \mid \overline{E_{T}\left(\lambda_{0}\right)} x_{\alpha}^{*}\right) \\
& \leqq \sup _{\alpha}\left\|x_{\alpha}\right\|^{2}=\sup _{\alpha} \varphi\left(T_{\alpha}\right)<\infty .
\end{aligned}
$$

This contradicts $\bar{\varphi}\left(\int_{0}^{\lambda_{\theta}} \lambda d \overline{E_{T}(\lambda)}\right)=\infty$. Consequently $\varphi$ is normal. Finally we shall show that $\varphi$ is semifinite. Since $\bar{\varphi}$ is semifinite, there exists a net $\left\{T_{\alpha}\right\}$ of $\left(\mathfrak{M}_{\varphi}\right)_{b}^{+}$such that $\bar{T}_{\alpha} \uparrow \bar{I}$. Let $T \in \mathfrak{U}^{+}$. By (6) we have

$$
T^{\frac{1}{2}} T_{\alpha} T^{\frac{1}{2}} \in \mathfrak{M}_{\varphi}^{+} \quad \text { and } \quad T^{\frac{1}{2}} T_{\alpha} T^{\frac{1}{2}} \uparrow T,
$$

and so $\varphi$ is semifinite.

Definition 4.3. The trace $\varphi$ of Theorem 4.2. is called the natural trace on $\mathfrak{U}^{+}$. 
Corollary 4.4. For every $A \in \mathfrak{A}$ and $x \in L_{2}^{\omega}\left(\mathscr{D}_{0}\right)$ we have

$$
\overline{\mathfrak{A}} L_{2}^{\omega}\left(\mathscr{D}_{0}\right) \subset L_{2}^{\omega}\left(\mathscr{D}_{0}\right) \quad \text { and } \quad \bar{A} \cdot \overline{\pi_{0}(x)}=\overline{\pi_{0}(\bar{A} x)} .
$$

In particular, we have

$$
\mathfrak{U} \mathfrak{M}_{\varphi} \subset \mathfrak{N}_{\varphi} \quad \text { and } \quad \mathfrak{U} \mathfrak{M}_{\varphi} \subset \mathfrak{M}_{\varphi}
$$

Proof. By Theorem 4.2.(7) we get $A=A_{0}+A_{1}, A_{0} \in \mathfrak{U}_{b}, A_{1} \in \mathfrak{M}_{\varphi}$, and so $\bar{A}=\overline{A_{0}}+\overline{\pi_{0}(y)}, y \in L^{\omega}\left(\mathscr{D}_{0}\right)$. Hence $\mathfrak{D}(\bar{A})=\mathfrak{D}\left(\overline{\pi_{0}(y)}\right) \supset L_{2}^{\omega}\left(\mathscr{D}_{0}\right)$ and we have

$$
\begin{aligned}
\bar{A} L_{2}^{\omega}\left(\mathscr{D}_{0}\right) & =\overline{A_{0}} L_{2}^{\omega}\left(\mathscr{D}_{0}\right)+\overline{A_{1}} L_{2}^{\omega}\left(\mathscr{D}_{0}\right) \\
& \subset L_{2}^{\omega}\left(\mathscr{D}_{0}\right),
\end{aligned}
$$

and

$$
\begin{aligned}
& \left.\bar{A} \cdot \overline{\pi_{0}(x)}=\overline{\left(A_{0}\right.}+\overline{\pi_{0}(y)}\right) \cdot \overline{\pi_{0}(x)} \\
& =\overline{A_{0}} \overline{\pi_{0}(x)}+\overline{\pi_{0}(y)} \cdot \overline{\pi_{0}(x)} \\
& =\overline{\pi_{0}\left(\overline{A_{0} x}\right)}+\overline{\pi_{0}\left(\overline{\pi_{0}(y)} x\right)} \\
& =\overline{\pi_{0}\left(\overline{A_{0}} x+\overline{A_{1}} x\right)} \\
& =\overline{\pi_{0}(\bar{A} x)} \text {. }
\end{aligned}
$$

Moreover, since $\overline{\mathfrak{N}_{\varphi}}=\overline{\mathfrak{A}} \cap L_{2}^{\omega}\left(\varphi_{0}\right)$ and $\overline{\mathfrak{M}_{\varphi}}=\overline{\mathfrak{A}} \cap L^{\omega}\left(\varphi_{0}\right)$, we have $\mathfrak{U} \mathfrak{N}_{\varphi} \subset \mathfrak{N}_{\varphi}$ and $\mathfrak{H} \mathfrak{M}_{\varphi} \subset \mathfrak{M}_{\varphi}$.

For every $A \in \mathfrak{U}$ putting

$$
\tilde{A} x=\bar{A} x, \quad x \in L_{2}^{\omega}\left(\mathscr{D}_{0}\right),
$$

$\tilde{A}$ is a linear operator on $L_{2}^{\omega}\left(\mathscr{D}_{0}\right)$ by Corollary 4.4 .. Let $\tilde{\mathfrak{U}}=$ $\{\tilde{A} ; A \in \mathfrak{A}\}$. Then we have

$$
\tilde{A} \tilde{B}=\tilde{A B}, \quad \lambda \tilde{A}=\tilde{\lambda A} \quad \text { and } \quad \tilde{A}^{*}=A^{*} / L_{2}^{\omega}\left(\mathscr{D}_{0}\right)=\widetilde{A^{*}}
$$

for every $A, B \in \mathfrak{A}$ and $\lambda \in(5$. We can easily show that $\mathfrak{A}$ equals the left $E W^{*}$-algebra $U\left(\Re\left(\mathscr{D}_{0}\right)\right)$ of a pure unbounded Hilbert algebra $\mathfrak{N}\left(\mathscr{D}_{0}\right)$. So, we obtain the following theorem.

THEOREM 4.5. Let $\mathscr{D}$ be a pure unbounded Hilbert algebra over $\mathscr{D}_{0}$ 
and let $\mathscr{E}$ be a pure unbounded Hilbert algebra over $\left(\mathscr{D}_{0}\right)_{b}$ containing $\mathscr{D}$. Let $\mathfrak{U}$ be a measurable $E W^{*}$-algebra on $\mathscr{E}$ such that $\frac{\mathscr{U}_{b}}{=\mathcal{U}_{0}}\left(\mathscr{D}_{0}\right)$ and $\overline{\mathfrak{U}} \supset \overline{\pi(\mathscr{D})}$. Then $\mathfrak{U}$ is regarded as the left $E W^{*}$-algebra $\mathscr{U}\left(\mathfrak{N}\left(\mathscr{D}_{0}\right)\right)$ of a pure unbounded Hilbert algebra $\mathfrak{N}\left(\mathscr{D}_{0}\right)$ over $\left(\mathscr{D}_{0}\right)_{b}$ containing $\mathscr{D}$.

Finally we shall show that an $E W^{*}$-algebra with a faithful normal semifinite trace is isomorphic to a left $E W^{*}$-algebra of a pure unbounded Hilbert algebra (Theorem 4.11). Let $\mathfrak{A}$ be an $E W^{*}$-algebra on $\mathfrak{D}$ and let $\varphi$ be a faithful trace on $\mathfrak{U}^{+}$. For each $S, T \in \mathfrak{N}_{\varphi}$ putting

$$
(\lambda(S) \mid \lambda(T))=\dot{\varphi}\left(T^{*} S\right)
$$

( | ) is an inner product on $\lambda\left(\mathfrak{N}_{\varphi}\right)$ and by, for each $S, T \in \mathfrak{N}_{\varphi}$ and $\alpha \in \mathfrak{C}$,

$$
\lambda(S)+\lambda(T)=\lambda(S+T), \quad \alpha \lambda(S)=\lambda(\alpha S),
$$

$\lambda\left(\mathfrak{N}_{\varphi}\right)$ is a pre-Hilbert space. Let $\mathfrak{K}_{\varphi}$ be the completion of $\lambda\left(\mathfrak{N}_{\varphi}\right)$. Let $\mathfrak{U}$ be a $\varphi$-measurable $E W^{*}$-algebra on $\mathfrak{D}$ and let $\varphi$ be a faithful normal semifinite trace on $\mathfrak{U}^{+}$satisfying $\mathfrak{U}\left(\mathfrak{N}_{\varphi}\right)_{b} \subset \mathfrak{N}_{\varphi}$.

LEMMA 4.6. The property " $\mathfrak{A}\left(\mathfrak{N}_{\varphi}\right)_{b} \subset \mathfrak{N}_{\varphi}$ " leads the property " $\mathfrak{H} \mathfrak{N}_{\varphi} \subset \mathfrak{N}_{\varphi}$ ".

Proof. Let $A \in \mathfrak{U}$ and $S \in \mathfrak{N}_{\varphi}$. Let $S=U|S|$ be the polar decomposition of $S$ and let $\overline{|S|}=\int_{0}^{\infty} \lambda d \overline{E_{S}(\lambda)}$ be the spectral resolution of $\overline{|S|}$. Since $\overline{|S|}$ is a $\bar{\varphi}$-restrictedly measurable operator, $\overline{E_{S}\left(\lambda_{0}\right)^{\perp}} \in \overline{\left(\mathfrak{M}_{\varphi}\right)_{b}}+$ for some $\lambda_{0}>0$, and so we have

$$
\begin{aligned}
A S=A U|S| & =A U\left(\int_{0}^{\lambda_{0}} \lambda d E_{S}(\lambda)+|S| E_{S}\left(\lambda_{0}\right)^{\perp}\right) \\
& =A U \int_{0}^{\lambda_{0}} \lambda d E_{S}(\lambda)+A S E_{S}\left(\lambda_{0}\right)^{\perp} \\
& \in \mathfrak{A}\left(\Re_{\varphi}\right)_{b} \subset \Re_{\varphi} .
\end{aligned}
$$

Lemma 4.7. Let $A \in \mathfrak{A} . \quad$ Then there exist $A_{0} \in \mathfrak{A}_{b}$ and $A_{1} \in \mathfrak{M}_{\varphi}$ such that

$$
A=A_{0}+A_{1}
$$

Proof. Let $A=U|A|$ be the polar decomposition of $A$ and let $\overline{|A|}=\int_{0}^{\infty} \lambda d \overline{E_{A}(\lambda)}$ be the spectral resolution. Since $\overline{|A|}$ is $\bar{\varphi}$ restrictedly measurable, $\overline{E_{A}\left(\lambda_{0}\right)^{\perp}} \in \overline{\left(\mathfrak{M}_{\varphi}\right)_{b}}{ }^{+}$for some $\lambda_{0}>0$. Putting 


$$
A_{0}=U\left(\int_{0}^{\lambda_{0}} \lambda d E_{A}(\lambda)\right) \text { and } A_{1}=A E_{A}\left(\lambda_{0}\right)^{\perp}
$$

$A_{0} \in \mathfrak{U}_{b}, A_{1} \in \mathfrak{U}\left(\mathfrak{M}_{\varphi}\right)_{b} \subset \mathfrak{M}_{\varphi}$ and $A=A_{0}+A_{1}$.

Lemma 4.8. The pre-Hilbert space $\lambda\left(\mathfrak{N}_{\varphi}\right)$ is a pure unbounded Hilbert algebra over $\lambda\left(\left(\mathfrak{N}_{\varphi}\right)_{b}\right)$.

Proof. We shall show that $\lambda\left(\left(\mathfrak{R}_{\varphi}\right)_{b}\right)$ is dense in $\lambda\left(\mathfrak{N}_{\varphi}\right)$. For each $T \in \mathfrak{N}_{\varphi}$ let $T=U|T|$ be the polar decomposition of $T$. Then $|T|=$ $U^{*} T \in \mathfrak{R}_{\varphi}^{+}$. Let $\overline{|T|}=\int_{0}^{\infty} \lambda d \overline{E_{T}(\lambda)}$ be the spectral resolution of $\overline{|T|}$. Putting

$$
\overline{S_{n}}=\int_{0}^{n} \lambda d \overline{E_{T}(\lambda)}
$$

$S_{n} \in\left(\Re_{\varphi}\right)_{b}^{+}$and $\left\{S_{n}\right\}$ converges $\sigma$-strongly to $|T|$, and so $S_{n}^{2} \uparrow|T|^{2}$ and since $\varphi$ is normal, we get

$$
\left\|\lambda\left(S_{n}\right)\right\|^{2}=\varphi\left(S_{n}^{2} \uparrow \varphi\left(|T|^{2}\right)=\|\lambda(|T|)\|^{2}\right.
$$

and

$$
\begin{aligned}
\left(\lambda(|T|) \mid \lambda\left(S_{n}\right)\right) & =\dot{\varphi}\left(|T| S_{n}\right) \\
& =\varphi\left(|T|^{\frac{1}{2}} S_{n}|T|^{\frac{1}{2}}\right) \uparrow \varphi\left(|T|^{2}\right)=\|\lambda(|T|)\|^{2},
\end{aligned}
$$

and hence

$$
\lim _{n \rightarrow \infty}\left\|\lambda\left(U S_{n}\right)-\lambda(T)\right\| \leqq \lim _{n \rightarrow \infty}\left\|\lambda\left(S_{n}\right)-\lambda(|T|)\right\|=0
$$

Therefore $\lambda\left(\left(\mathfrak{N}_{\varphi}\right)_{b}\right)$ is dense in $\lambda\left(\mathfrak{N}_{\varphi}\right)$. Since $\bar{\varphi}$ is a faithful normal semifinite trace on $\overline{\mathfrak{A}}_{b}^{+}, \lambda\left(\overline{\left(\mathfrak{N}_{\varphi}\right)_{b}}\right)=\lambda\left(\mathfrak{N}_{\bar{\varphi}}\right)$ is a maximal Hilbert algebra, and so we can easily show that $\lambda\left(\left(\mathfrak{N}_{\varphi}\right)_{b}\right)$ is a maximal Hilbert algebra. For every $S, T \in \mathfrak{N}_{\varphi}$ we define the operations on $\lambda\left(\mathfrak{N}_{\varphi}\right)$ as follows;

$$
\begin{array}{ll}
\lambda(S) \lambda(T)=\lambda(S T), & \alpha \lambda(S)=\lambda(\alpha S), \\
\lambda(S)^{*}=\lambda\left(S^{*}\right), & (\lambda(S) \mid \lambda(T))=\dot{\varphi}\left(T^{*} S\right) .
\end{array}
$$

Then it is not difficult to show that $\lambda\left(\mathfrak{N}_{\varphi}\right)$ is an unbounded Hilbert algebra over $\lambda\left(\left(\mathfrak{N}_{\varphi}\right)_{b}\right)$. Finally we shall show that $\lambda\left(\mathfrak{N}_{\varphi}\right)$ is pure. By 
Lemma 4.7. every element $A$ of $\mathfrak{A}$ is represented by $A=A_{0}+A_{1}$, $A_{0} \in \mathfrak{U}_{b}, \quad A_{1} \in \mathfrak{M}_{\varphi} . \quad$ If $\quad A \in \mathfrak{U}-\mathfrak{A}_{b}$, then $A_{1} \in \mathfrak{M}_{\varphi}-\left(\mathfrak{M}_{\varphi}\right)_{b}$, and so $\lambda\left(\left(\mathfrak{N}_{\varphi}\right)_{b}\right) \neq \lambda\left(\mathfrak{N}_{\varphi}\right)$ and $\lambda\left(\left(\mathfrak{N}_{\varphi}\right)_{b}\right)$ is a maximal Hilbert algebra. Therefore $\lambda\left(\mathfrak{N}_{\varphi}\right)$ is pure.

Lemma 4.9. For every $A \in \mathfrak{A}$ putting

$$
\Psi(A) \lambda(T)=\lambda(A T), \quad T \in \mathfrak{N}_{\varphi},
$$

$\Psi(A)$ is a linear operator on $\lambda\left(\mathfrak{N}_{\varphi}\right) . \quad \Psi(\mathfrak{U})$ is a measurable $E W^{*}$-algebra on $\lambda\left(\mathfrak{N}_{\varphi}\right)$ such that $\overline{\Psi(\mathfrak{U})_{b}}=\overline{\Psi\left(\mathfrak{U}_{b}\right)}=\mathscr{U}_{0}\left(\lambda\left(\left(\mathfrak{N}_{\varphi}\right)_{b}\right)\right)$ and $\overline{\Psi(\mathfrak{U})} \supset \overline{\pi\left(\lambda\left(\mathfrak{N}_{\varphi}\right)\right)}$ and $\Psi$ is an isomorphism of $\mathfrak{A}$ onto $\Psi(\mathfrak{U})$.

Proof. By Lemma 4.6. $\mathfrak{U} \mathfrak{N}_{\varphi} \subset \mathfrak{N}_{\varphi}$, and so $\Psi(A)$ is a linear operator on $\lambda\left(\mathfrak{N}_{\varphi}\right)$. For every $S \in \mathfrak{R}_{\varphi}$ we have $\Psi(S)=\pi(\lambda(S))$, where $\pi$ is the left regular representation of the pure unbounded Hilbert algebra $\lambda\left(\mathfrak{N}_{\varphi}\right)$. We shall show $\Psi(\mathfrak{H})_{b}=\Psi\left(\mathfrak{H}_{b}\right)$. Clearly we have $\Psi\left(\mathfrak{U}_{b}\right) \subset \Psi(\mathfrak{H})_{b}$. Conversely let $\Psi(A) \in \Psi(\mathfrak{U})_{b}$. By Lemma 4.7. $A=A_{0}+A_{1}, \quad A_{0} \in \mathfrak{A}_{b}$, $A_{1} \in \mathfrak{M}_{\varphi}$, and so $\Psi\left(A_{1}\right)=\pi\left(\lambda\left(A_{1}\right)\right) \in \Psi\left(\mathfrak{M}_{\varphi}\right)_{b}$. Since $\lambda\left(\left(\mathfrak{N}_{\varphi}\right)_{b}\right)$ is a maximal Hilbert algebra, $\lambda\left(A_{1}\right) \in \lambda\left(\left(\mathfrak{N}_{\varphi}\right)_{b}\right)$, i.e., $A_{1} \in\left(\mathfrak{N}_{\varphi}\right)_{b}$. Therefore $A=$ $A_{0}+A_{1} \in \mathfrak{A}_{b}$, and so $\Psi(A) \in \Psi\left(\mathfrak{U}_{b}\right)$. By the theory of von Neumann algebras, $\overline{\Psi\left(\mathfrak{A}_{b}\right)}=\mathcal{U}_{0}\left(\lambda\left(\left(\mathfrak{N}_{\varphi}\right)_{b}\right)\right)$. Moreover it is easy to show that $\Psi(\mathfrak{A}) \supset \Psi\left(\mathfrak{N}_{\varphi}\right)=\pi\left(\lambda\left(\mathfrak{N}_{\varphi}\right)\right)$ and $\Psi$ is an isomorphism of $\mathfrak{A}$ onto $\Psi(\mathfrak{U})$. Since $\mathfrak{U}$ is $\varphi$-measurable, we can easily show that $\Psi(\mathfrak{U})$ is measurable.

LEMMA 4.10. Let $\psi$ be the natural trace on $\Psi(\mathfrak{H})^{+}$. Then we have

$$
\varphi(A)=\psi(\Psi(A)), \quad A \in \mathfrak{U}^{+} .
$$

Proof. By the definition of the natural trace $\psi$ we get

$$
\mathfrak{M}_{\psi}^{+}=\pi\left(\lambda\left(\mathfrak{M}_{\varphi}^{+}\right)\right)=\Psi\left(\mathfrak{M}_{\varphi}^{+}\right)
$$

and moreover for every $A \in \mathfrak{M}_{\varphi}^{+}$

$$
\varphi(A)=\left\|\lambda\left(A^{\frac{1}{2}}\right)\right\|^{2}=\psi(\pi(\lambda(A)))=\psi(\Psi(A)) .
$$

By Lemma 4.6. $\sim 4.10$, and Theorem 4.5. we obtain the following theorem.

THEOREM 4.11. Let $\mathfrak{A}$ be an $E W^{*}$-algebra and let $\varphi$ be a faithful normal semifinite trace on $\mathfrak{A}^{+}$. Suppose that $\mathfrak{A}$ is a $\varphi$-measurable 
$E W^{*}$-algebra and $\mathfrak{A}\left(\mathfrak{N}_{\varphi}\right)_{b} \subset \mathfrak{N}_{\varphi}$. Then $\lambda\left(\mathfrak{N}_{\varphi}\right)$ is a pure unbounded Hilbert algebra over $\lambda\left(\left(\mathfrak{N}_{\varphi}\right)_{b}\right)$ and putting

$$
\Psi(A) \lambda(S)=\lambda(A S), \quad S \in \mathfrak{N}_{\varphi}
$$

for every $A \in \mathfrak{A}, \Psi(A)$ is a linear operator on $\lambda\left(\mathfrak{N}_{\varphi}\right)$. The isomorphism $\Psi$ is extended to an isomorphism $\Phi$ of $\mathfrak{A}$ onto the left $E W^{*}$-algebra $U\left(\lambda\left(\Re_{\varphi}\right)\right)$ of $\lambda\left(\mathfrak{N}_{\varphi}\right)$. Let $\psi$ be the natural trace on $\Phi(\mathfrak{A})^{+}$. Then $\varphi=\psi \circ \Phi$.

\section{REFERENCES}

1. R. Arens, The space $L^{\omega}$ and convex topological rings, Bull. Amer. Math. Soc., 52 (1946), 931-935.

2. G. R. Allan, On a class of locally convex algebras, Proc. London Math. Soc., (3) 17 (1967), 91-114.

3. W. Ambrose, The $L^{2}$-system of a unimodular group, Trans. Amer. Math. Soc., 65 (1949), $27-48$.

4. J. Dixmier, Algèbres quasi-unitaire, Comment. Math. Helv., 26 (1952), 275-322.

5. L Les Algèbres D'operateurs dans L'espace Hilbertien, Gausthier-Villars, Paris, 2é edition (1969).

6. P. G. Dixon, Generalized B*-algebras, Proc. London Math. Soc., (3) 21 (1970), 693-715.

7. _- Unbounded operator algebras, Proc. London Math. Soc., (3) 23 (1971), 53-69.

8. N. Dunford and J. Schwartz, Linear operators vol II, New York; Interscience Pub. (1963).

9. R. Godement, Théorie des caractères. I. Algèbres unitaires, Ann. Math., 59 (1954), 47-69.

10. A. Inoue, On a class of unbounded operator algebras, Pacific J. Math., 65 (1976), 77-95.

11. T. Ogasawara and K. Yoshinaga, A noncommutative theory of integration for operators, J. Sci. Hiroshima Univ., 18 (3) (1955), 311-347.

12. R. Pallu de La Barrière, Algèbres unitaires et espaces d'Ambrose, Ann. Éc. Norm. Sup., 70 (1953), 381-401.

13. R. T. Powers, Self-adjoint algebras of unbounded operators, Commun. Math. Phys., 21 (1971), 85-124.

14. I. E. Segal, A noncommutative extension of abstract integration, Ann. Math., 57 (1953), 401-457.

15. A. Weil, L'Intégration dans les Groupes Topologiques et Ses Applications, 2e éd. Act. Sc. Ind., no 1145. Hermann, Paris, 1953.

Received February 10, 1976.

FUKUOKA UNIVERSITY 



\section{PACIFIC JOURNAL OF MATHEMATICS}

\section{EDITORS}

RICHARI) ARENS (Managing Editor)

University of California

Los Angeles, CA 90024

R. A. Beaumiont

University of Washington

Seattle, WA 98105

\section{J. DugunduI}

Department of Mathematics University of Southern California Los Angeles, CA 90007

D. Gilbarg and J. Milgram Stanford University

Stanford, CA 94305

\section{ASSOCIATE EDITORS}

E. F. BECKENBACH
B. H. NEUMANN

F. Wolf

K. YoshidA

\section{SUPPORTING INSTITUTIONS}

UNIVERSITY OF BRITISH COLUMBIA CALIFORNIA INSTITUTE OF TECHNOLOGY

UNIVERSITY OF CALIFORNIA

MONTANA STATE UNIVERSITY

UNIVERSITY OF NEVADA

NEW MEXICO STATE UNIVERSITY

OREGON STATE UNIVERSITY

UNIVERSITY OF OREGON

OSAKA UNIVERSITY

\author{
UNIVERSITY OF SOUTHERN CALIFORNIA \\ STANFORD UNIVERSITY \\ UNIVERSITY OF HAWAII \\ UNIVERSITY OF TOKYO \\ UNIVERSITY OF UTAH \\ WASHINGTON STATE UNIVERSITY \\ UNIVERSITY OF WASHINGTON \\ AMERICAN MATHEMATICAL SOCIETY
}

The Supporting Institutions listed above contribute to the cost of publication of this Journal, but they are not owners or publishers and have no responsibility for its contents or policies.

Mathematical papers intended for publication in the Pacific Journal of Mathematics should be in typed form or offset-reproduced (not dittoed), double spaced with large margins. Underline Greek letters in red, German in green, and script in blue. The first $p: 1<$ graph or two must be capable of being used separately as a synopsis of the entire paper. Items of the biblography should not be cited there unless absolutely necessary, in which case they must he identified by author and Journal, rather than by item number. Manuscripts, in duplicate, may be sent to any one of the four editors. Please classify according to the scheme of Math. Reviews, Index to Vol. 39. All other communications should be addressed to the managing editor, or Elaine Barth, University of California, Los Angeles, California, 90024.

100 reprints are provided free for each article, only if page charges have been substantially paid. Additional copies may be obtained at cost in multiples of 50 .

The Pacific Journal of Mathematics is issued monthly as of January 1966. Regular subscription rate: $\$ 72.00$ a year (6 Vols., 12 issues). Special rate: $\$ 36.00$ a year to individual members of supporting institutions.

Subscriptions, orders for back numbers, and changes of address should be sent to Pacific Journal of Mathematics, 103 Highland Boulevard, Berkeley, California, 94708.

PUBLISHED BY PACIFIC JOURNAL OF MATHEMATICS, A NON-PROFIT CORPORATION Printed at Jerusalem Academic Press, POB 2390, Jerusalem, Israel.

\section{Copyright (C) 1976 Pacific Journal of Mathematics} All Rights Reserved 


\section{Pacific Journal of Mathematics}

\section{Vol. 66, No. 2 December, 1976}

Gerald A. Beer, Tax structures whose progressivity is inflation neutral..... 305

William M. Cornette, A generalization of the unit interval............. 313

David E. Evans, Unbounded completely positive linear maps on

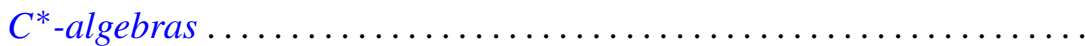

Hector O. Fattorini, Some remarks on convolution equations for

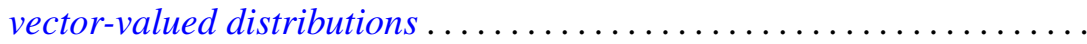

Amassa Courtney Fauntleroy, Automorphism groups of unipotent groups of

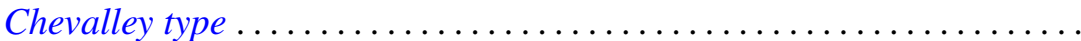

Christian C. Fenske and Heinz-Otto Peitgen, On fixed points of zero index in

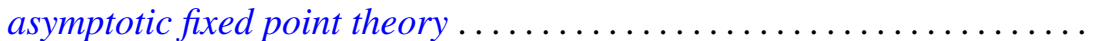

Atsushi Inoue, On a class of unbounded operator algebras. II ............

Herbert Meyer Kamowitz, The spectra of endomorphisms of algebras of

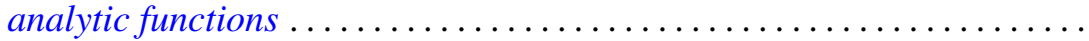

Jimmie Don Lawson, Embeddings of compact convex sets and locally compact cones ....................................

William Lindgren and Peter Joseph Nyikos, Spaces with bases satisfying certain order and intersection properties .....................

Emily Mann Peck, Lattice projections on continuous function spaces ...... 477

Morris Marden and Peter A. McCoy, Level sets of polynomials in $n$ real variables...

Francis Joseph Narcowich, An imbedding theorem for indeterminate Hermitian moment sequences......................

John Dacey O'Neill, Rings whose additive subgroups are subrings ...

Chull Park and David Lee Skoug, Wiener integrals over the sets bounded by sectionally continuous barriers .....................

Vladimir Scheffer, Partial regularity of solutions to the Navier-Stokes equations.

Eugene Spiegel and Allan Trojan, On semi-simple group algebras. II

Katsuo Takano, On Cameron and Storvick's operator valued function space integral 